\title{
Back Massage to Decrease State Anxiety, Cortisol Level, Blood Prsessure, Heart Rate and Increase Sleep Quality in Family Caregivers of Patients with Cancer: A Randomised Controlled Trial
}

\author{
Rukiye Pinar ${ }^{1 *}$, Fisun Afsar ${ }^{2}$
}

\begin{abstract}
Background: The objective of this study was to evaluate the effect of back massage on the anxiety state, cortisol level, systolic/diastolic blood pressure, pulse rate, and sleep quality in family caregivers of patients with cancer. Materials and Methods: Forty-four family caregivers were randomly assigned to either the experimental or control group (22 interventions, 22 controls) after they were matched on age and gender. The intervention consisted of back massage for 15 minutes per day for a week. Main research outcomes were measured at baseline (day I) and follow-up (day 7). Unpaired t-test, paired $t$ test and chi-square test were used to analyse data. Results: The majority of the caregivers were women, married, secondary school educated and housewife. State anxiety $(\mathbf{p}<0.001)$, cortisol level $(\mathbf{p}<0.05)$, systolic/diastolic blood pressure $(\mathbf{p}<0.001, \mathbf{p}<0.01$ respectively), and pulse rate $(\mathbf{p}<0.01)$ were significantly decreased, and sleep quality $(p<0.001)$ increased after back massage intervention. Conclusions: The study results show that family caregivers for patients with cancer can benefit from back massage to improve state anxiety, cortisol level, blood pressure and heart rate, and sleep quality. Oncology nurses can take advantage of back massage, which is non-pharmacologic and easily implemented method, as an independent nursing action to support caregivers for patients with cancer.
\end{abstract}

Keywords: Anxiety - blood pressure - cortisol - cancer family caregivers - heart rate - massage - massage therapy

Asian Pac J Cancer Prev, 16 (18), 8127-8133

\section{Introduction}

Over the past half century, awareness on complex care needs of cancer patients has been increased. As a result of this, increased numbers of family members who take care, help and support cancer patients through the disease have been involved in health care systems (Tamayo et al., 2010).

Caring for a patient with cancer is a demanding, overwhelming and uncompensating process because family caregivers have to shoulder the responsibilities for almost everything including providing physical care and emotional support to patients, helping symptom management, in addition, performing routine households including cleaning, cooking, shopping etc. These demanding tasks have a major impact on a caregiver's life although the roles of the family caregivers are often invisible and unrecognized (Lambert et al., 2013).

Negative psychological and physiological health consequences of caregiving including stress (Palos et al., 2011; Li and Loke, 2013; Pollock et al., 2013; Lund et al., 2014); anxiety (Cora et al., 2012; Lambert et al., 2013; Li and Loke, 2013; Wasner et al., 2013); sleep disturbances (Stenberg et al., 2010; Usta Yesilbalkan et al., 2010; Palos et al., 2011; Cora et al., 2012; Li and Loke, 2013; Pollock et al., 2013; Morris et al., 2015); increased blood pressure (BP) and heart rate (HR) (Cora et al., 2012) and decreased quality of life (QOL) (Usta Yesilbalkan et al., 2010; Turkoglu and K1lıc, 2012; Li and Loke, 2013; Butow et al., 2014, Zhu et al., 2014); which all are associated with increased stress or strain, have been reported in earlier studies (Palos et al., 2011; Li and Loke, 2013).

In a cross sectional study with a large sample consisting of 590 caregivers from randomly selected cancer patients, it was shown that $59 \%$ of caregivers experienced stress (Lund et al., 2014).

Anxiety is a common health problem as well. Wasner et al. (2013) reported that $45 \%$ of caregivers of primary malignant brain tumour patients had anxiety. In another study (Lambert et al., 2013), a third of caregivers had anxiety, exceeding community norms. Mitchell et al. study (2013) suggested that anxiety is most likely to be a problem in long-term cancer survivors' spouses rather than depression.

Higher subjective anxiety leads to an increase in cortisol levels. State anxiety levels increase as an initial response to stress. When anxiety reaches plateau level, 
it remains stable, and eventually, the nature of stress response changes and cortisol is released (Cora et al., 2012). It is well known that cortisol is significantly increased by psychological stressors, especially in chronic stressful events. In the presence of increased cortisol, the sympathetic function of autonomic nervous system increases, then BP and HR increase (Ouchi et al., 2006).

Although elevated BP and HR are both key measures of cardiovascular health risk, as far as our present knowledge extends, there is only one study that has been conducted on cardiovascular impact of family caregiving for cancer patients. This pilot study showed that family caregivers had higher level of state anxiety, elevated BP and HR, and more sleep dysfunctions than the controls (Cora et al., 2012).

Sleep is essential for optimum mental (Pollock et al., 2013) and physical health (Northouse et al., 2012), as well as for quality of life (Usta Yesilbalkan et al., 2010). The previous studies showed that the most caregivers of patients with cancer had sleep-related problems. For example, Pollock et al. (2013) showed that parents of childhood cancer and brain tumour survivors have higher level poor sleep quality than their controls. In a literature review, including 192 articles published between 1990 and 2008, sleep disturbance is shown among the most prevalent problems (Stenberg et al., 2010). In Morris et al study (2015) insomnia symptoms were reported by $62.9 \%$ of caregivers.

In sum, negative consequences of caregiving, such as stress/strain (Palos et al., 2011; Li and Loke, 2013; Pollock et al., 2013; Lund et al., 2014); anxiety (Cora et al., 2012; Lambert et al., 2013; Li and Loke, 2013; Wasner et al., 2013); poor sleep quality (Stenberg et al., 2010; Usta Yesilbalkan et al., 2010; Palos et al., 2011; Cora et al., 2012; Li and Loke, 2013; Pollock et al., 2013; Morris et al., 2015); and elevated BP and HR (Cora et al., 2012) confirm that cancer caregiving is burdensome.

Furthermore, a caregiver's burden also effects caregiver's ability to provide care for the patient and might resulted in unmet patient's needs (Given and Sherwood, 2006). For that reason, we believe that there is a crucial need for using supportive interventions for this vulnerable group. To our knowledge, unfortunately, there are only several studies on supportive interventions for caregivers of patients with cancer.

MacDonald (1998) found that massage will be useful to reduce physical and emotional stress and to increase sleep quality in hospice settings. Mackereth et al. (2005) showed positive effects of chair massage on caregivers at a major cancer hospital in United Kingdom. In Iwasaki's study (2005), 68 women with hospitalized children received hand and shoulder massage compared to 20 mothers with healthy children. In a study conducted by Rexilius et al. (2002), 33 caregivers of patients undergoing autologous hematopoietic stem cell transplant were divided into a massage, a healing touch and a control group. In Goodfellow's study (2003), 42 spouses of patients with cancer were randomly assigned to either the therapeutic back massage or the control group. The results of those studies showed that massage reduced tensionanxiety (Rexilius et al., 2002; Iwasaki, 2005) and stress, and had positive effects on mood (Goodfellow, 2003). In addition, Lengacher et al. (2012) found that back massage will be a beneficial intervention to reduce cortisol levels, BP and HR, and to improve sleep quality.

These results show that the need to understand the caregivers' experiences has gained more importance. In addition, more assistive interventions for caregivers need to be implemented. The back massage, which is a complementary, non-pharmacologic and easily implemented method, as an independent nursing function can be used to support caregivers for patients with cancer. Therefore, the current study aimed to examine the effectiveness of back massage on state anxiety, cortisol level, BP, HR and sleep quality in caregivers of patients with cancer.

\section{Materials and Methods}

\section{Setting and sample}

The study was conducted in three inpatient oncology clinics at a state hospital between March 2012 and September 2012.

Caregivers were eligible to participate if they: were $\geq 18$ years of age; were primary family caregivers who mainly provided support to the patient; able to complete questionnaires; and agreed to participate in the study. Exclusion criteria were pre-existing neck and lower back hernia, dermatological problems, infection, hypertension, renal impairment, cardiothoracic problems, or any condition that would interfere with the massage intervention. We also excluded caregivers who were pregnant, who were in a menstrual cycle, and had acute pain. Forty-four caregivers met the inclusion criteria among 136.

Those caregivers were randomly assigned to either the intervention or control group (22 interventions, 22 controls) after they were matched on age and gender. Blocked randomization method was applied to achieve balance in intervention and control groups (Figure 1).

Written informed consent was obtained from participants before the study. All procedures in the study were approved by the university's Institutional Ethics Committee (Study reference number: MARYÇ-2012-0166).

\section{Outcome variables}

State anxiety. State anxiety level was measured by using the State-Trait Anxiety Inventory (STAI) (Spielberger et al., 1983). The STAI consists of two separate scales for measuring state (STAI-s) and trait anxiety (STAI-t). We used STAI-s part of STAI to measure state anxiety. Scores for the STAI-s range from 20 to 80 , with higher scores reflecting more anxiety. Scores 0-19 correspond to "no anxiety", 20-39 to "a low anxiety", 4059 to "moderate anxiety" and 60 to 79 to "higher anxiety", and 80 and over to "panic or crisis". Validation study of the Turkish STAI-s was done by Oner and Le Compte (1985).

Cortisol level. Cortisol is a sensitive indicator reflecting the stress response of the body. In this study, venous whole blood sample was collected from each caregiver to evaluate effects of massage on cortisol level. 
Back Massage Decreases Anxiety, Blood Pressure, Heart Rate and Increases Sleep Quality in Cancer Patient Caregivers

The collected blood was centrifuged for $10 \mathrm{~min}$ at 3,000 rpm at $4^{\circ} \mathrm{C}$ to remove plasma. Then we pipetted plasma into tubes and stored it at $-80^{\circ} \mathrm{C}$. Plasma samples were later assayed in duplicate using commercially available kits (Modular Analytics E170, Roche Diagnostics, Mannheim, Germany). Acceptable reference cortisol levels for the evening were between $2.3-11.9 \mathrm{mcg} / \mathrm{dl}$ (61-317 nmol/L).

$\mathrm{BP}$ and HR. BP and HR were measured by using a calibrated digital BP machine in sitting position after resting for at least 5 minutes. Readings were taken twice with five minute intervals between measurements and the average of two measurements was calculated.

Sleep quality. To assess sleep quality, we used Pittsburgh Sleep Quality Index (PSQI), (Buysse et al., 1989). The PSQI consists of 19 items generating seven "component" scores: subjective sleep quality, sleep latency, sleep duration, habitual sleep efficiency, sleep disturbances, use of sleeping medication, and daytime dysfunction. Each component score ranges from 0 to 3 , the sum of the scores from all seven components determines sleep quality. The global PSQI score ranges from 0 to 21. The total PSQI score of 0-4.99 indicates good sleep quality; $\geq 5$ indicates poor sleep quality. A Turkish version of the PSQI is available (Agargun et al., 1996).

Descriptive variables. Descriptive variables were measured by a general information form at the baseline enrollment appointment, including age, sex, education, working status, marital status, having children, income, relationship with the patient, and whether they were living together with the patient or not.

\section{Protocol}

Before the massage, the caregivers in the intervention group rested in a chair for 10 minutes in a silence room

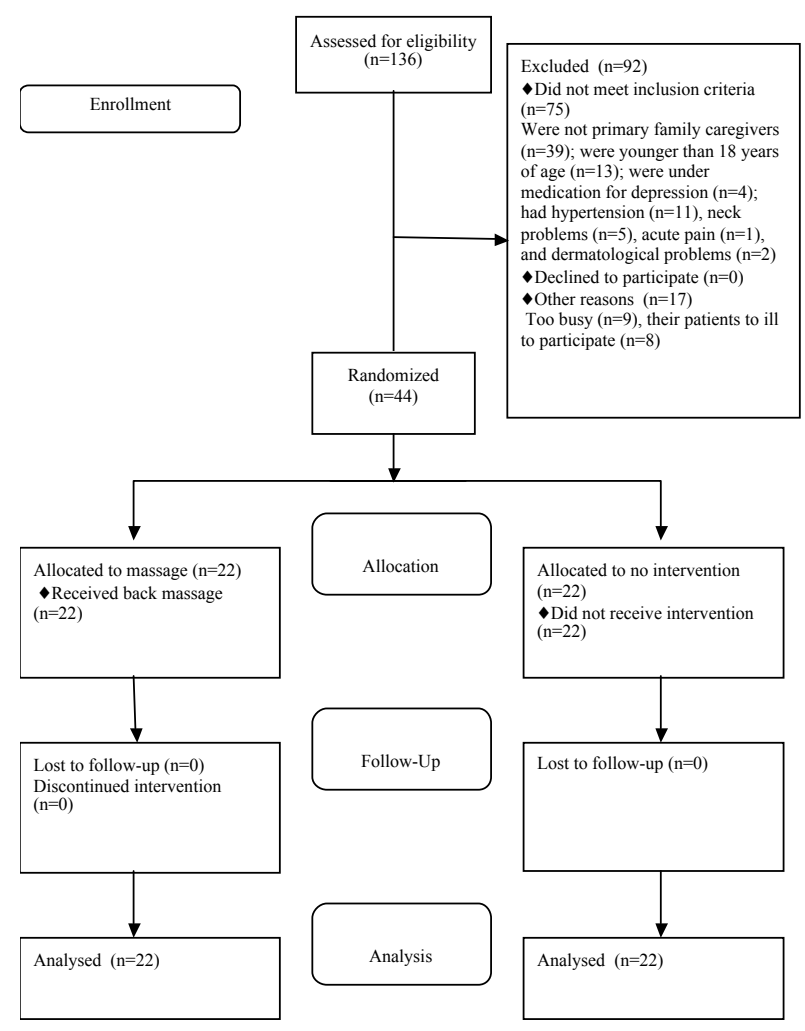

Figure 1. Consort Flow Diagram at the oncology clinics. A "do not disturb, massage in progress" sign was posted on the room's door. The caregivers then received daily 15 minutes back massage at medium pressure for a week (seven sessions) by one of the authors who has been educated for back massage. Massage was carried out in the evening times between 5.00-8.00 p.m. Very little conversation occurred during the massage, when necessary.

Each massage session, consisting of a combination of effleurage (rhythmic, gliding strokes), petrissage (gentle kneading), friction (rhythmic pressing), and tapotement (quick, striking movements or beating), started on the dorsal-lumbar region, followed by the back, scapulas, shoulders, neck, and scalp (from frontal area to occipital area). In each session, pressure was applied using sliding, and circular movements and straight line movements to warm up and massage the skin. Non-aromatic oil (baby oil) was used for lubrication.

The control group rested quietly in a different silence room. They were not allowed any activities that may affect outcome parameters, including listening to music, watching television, or exercising.

On Day 1, all caregivers in the intervention and control groups were administrated the STAI-s and the PSQI; and blood samples for cortisol test were collected. Same procedures were repeated on Day 7. Blood samples for cortisol test were collected immediately before the administration of massage on Day 1 and within 10 minutes after the administration of massage on Day 7 in the intervention group. BP and HR were taken in only intervention group, before and after massage on Day I and Day 7. Blood samples were collected in both groups between 5.00 p.m and 8.00 p.m.

\section{Statistical analysis}

We used Statistical Package for the Social Sciences (SPSS)-version 16.0 to perform statistical analyses. Descriptive statistics were used to describe the caregivers and assess the distribution of data. Baseline data for intervention and massage group were compared by using the unpaired t-test and chi-square test. Changes in anxiety level, cortisol level, BP, HR and sleep quality before and after massage in the intervention group at Day 1 and Day 7 were analyzed by paired t test and chi-square test. Comparisons in the control group at Day 1 and Day 7 were analysed by paired t test and chi-square test as well. All statistical tests were considered significant as $\mathrm{p}$ value lower than 0.05 .

\section{Results}

\section{Characteristics of family caregivers}

The characteristics of family caregivers in the intervention and control groups are presented in Table 1. Caregivers' average age was 42.3. The majority of them were women who had secondary school education, had not being working, had moderate level of income, and were living together with the patients. All caregivers were married and had children. More than half of the caregivers in the massage group were spouses of cancer patients, while in the control group, the caregivers were parents 
Rukiye Pinar and Fisun Afsar

(mother or father) of the patients. The characteristics of the family caregivers were not differed significantly between massage and control groups.

\section{Effects of massage}

Table 2 shows the effects of massage on state anxiety, cortisol level and sleep quality, while Table 3 shows the effects of massage on BP and HR.
As shown in the Table 2 and Table 3, back massage resulted in positive changes in state anxiety, cortisol level, BPs, HR, and sleep quality.

In the present study, all caregivers in the massage group had moderate level of anxiety, while 31.8 percent of the control group had low level of anxiety, 59.1 percent had moderate level of anxiety, and 9.1 percent had high level of anxiety. At the final evaluation, 3 caregivers (13.6\%)

Table 1. Characteristics of Family Caregivers ( $N=44)$

\begin{tabular}{|c|c|c|c|}
\hline & Massage Group $(\mathrm{N}=22)$ & Control Group $(\mathrm{N}=22)$ & Significance \\
\hline Age, mean year $\pm S D,($ range $)$ & $\begin{array}{c}41.72 \pm 8.31 \\
(\text { Range }=24-60 \mathrm{yrs}) \\
\mathrm{n}(\%)\end{array}$ & $\begin{array}{c}42.90 \pm 9.22 \\
(\text { Range }=25-56 \mathrm{yrs}) \\
\mathrm{n}(\%)\end{array}$ & $\begin{array}{c}\mathrm{t}=0.45 \\
\mathrm{P}=0.322\end{array}$ \\
\hline \multicolumn{4}{|l|}{ Sex } \\
\hline $\begin{array}{l}\text { Female } \\
\text { Male }\end{array}$ & $\begin{array}{r}14(63.6 \%) \\
8(36.4 \%)\end{array}$ & $\begin{array}{r}14(63.6 \%) \\
8(36.4 \%)\end{array}$ & $\begin{array}{l}\mathrm{X}^{2}=0.00 \\
\mathrm{P}=0.625\end{array}$ \\
\hline \multicolumn{4}{|l|}{ Education } \\
\hline Primary school (5 yrs) & $\begin{array}{l}8(36.4 \%) \\
14(636 \%)\end{array}$ & $11(50 \%)$ & $\begin{array}{l}x^{2}=0.83 \\
P=0.464\end{array}$ \\
\hline \multicolumn{4}{|l|}{ Working status } \\
\hline $\begin{array}{l}\text { Day time } \\
\text { Not working }\end{array}$ & $\begin{array}{c}5(22.8 \%) \\
17(77.3 \%)\end{array}$ & $\begin{array}{c}6(27.3 \%) \\
16(72.7 \%)\end{array}$ & $\begin{array}{l}\mathrm{X}^{2}=0.12 \\
\mathrm{P}=0.834\end{array}$ \\
\hline \multicolumn{4}{|l|}{ Marrital status } \\
\hline Married & $22(100 \%)$ & $22(100 \%)$ & $\begin{array}{l}\mathrm{X}^{2}=0.00 \\
\mathrm{P}=1.000\end{array}$ \\
\hline \multicolumn{4}{|l|}{ Having children } \\
\hline Yes & $22(100 \%)$ & $22(100 \%)$ & $\begin{array}{l}x^{2}=0.00 \\
P=1.000\end{array}$ \\
\hline \multicolumn{4}{|l|}{ Income } \\
\hline Poor & $3(13.6 \%)$ & $3(13.6 \%)$ & $x^{2}=1.58$ \\
\hline Moderate & $14(63.6 \%)$ & $17(77.3 \%)$ & $\mathrm{P}=0.456$ \\
\hline Good & $5(22.8 \%)$ & $2(9.1 \%)$ & \\
\hline \multicolumn{4}{|l|}{ Relationship } \\
\hline Spouse & $13(59.1 \%)$ & $10(45.5 \%)$ & $\mathrm{x}^{2}=0.82$ \\
\hline Mother-father & $9(46.9 \%)$ & $12(54.5 \%)$ & $\mathrm{P}=0.273$ \\
\hline \multicolumn{4}{|l|}{ Living together with patients } \\
\hline Yes & $19(86.4 \%)$ & $14(63.6 \%)$ & $x^{2}=3.03$ \\
\hline No & $3(13.6 \%)$ & $8(36.4 \%)$ & $\mathrm{P}=0.081$ \\
\hline
\end{tabular}

Table 2. Effects of Massage on State Anxiety, Cortisol Level and, Sleep Quality

\begin{tabular}{|c|c|c|c|c|c|c|}
\hline & \multicolumn{3}{|c|}{ Massage Group $(\mathrm{N}=22)$} & \multicolumn{3}{|c|}{ Control Group $(\mathrm{N}=22)$} \\
\hline & Day I & Day 7 & Significance & Day I & Day 7 & Significance \\
\hline \multicolumn{7}{|l|}{ STAI } \\
\hline Low & & $3(13.6 \%)$ & & $7(31.8 \%)$ & $7(31.8 \%)$ & \\
\hline Moderate & $22(100 \%)$ & $19(86.4 \%)$ & $\mathrm{x}^{2}=3.22(\mathrm{P}=0.073)$ & $13(59.1 \%)$ & $13(59.1 \%)$ & $\mathrm{x}^{2}=0.00$ \\
\hline High & & & & $2(9.1 \%)$ & $2(9.1 \%)$ & $(\mathrm{P}=1.000)$ \\
\hline $\begin{array}{l}\text { Low anxiety } \\
(\text { Mean } \pm \text { SD) }\end{array}$ & & & & $31.14 \pm 3.84$ & $32.57 \pm 4.79$ & $\mathrm{t}=1.69,(\mathrm{P}=0.140)$ \\
\hline $\begin{array}{l}\text { Moderate anxiety } \\
(\text { Mean } \pm \text { SD })\end{array}$ & $49.68 \pm 7.22$ & $45.68 \pm 6.67$ & $\mathrm{t}=11.92(\mathrm{P}=0.000) * * *$ & $49.07 \pm 6.21$ & $51.69 \pm 6.70$ & $\mathrm{t}=2.06,(\mathrm{P}=0.062)$ \\
\hline $\begin{array}{l}\text { High anxiety } \\
(\text { Mean } \pm \text { SD) }\end{array}$ & & & & $66.50 \pm 4.94$ & $65.00 \pm 11.31$ & $\mathrm{t}=0.33,(\mathrm{P}=0.795)$ \\
\hline $\begin{array}{l}\text { Cortisol level } \\
(\text { Mean } \pm \text { SD) }\end{array}$ & $7.43 \pm 1.21$ & $7.37 \pm 1.34$ & $\mathrm{t}=2.83(\mathrm{P}=0.01)^{*}$ & $7.31 \pm 0.91$ & $7.32 \pm 0.91$ & $\mathrm{t}=1.34,(\mathrm{P}=0.204)$ \\
\hline \multicolumn{7}{|l|}{ PSQI } \\
\hline$<5$ & $5(22.8 \%)$ & $8(36.4 \%)$ & $\mathrm{x}^{2}=0.98,(\mathrm{P}=0.108)$ & $5(22.8 \%)$ & $5(22.8 \%)$ & $x^{2}=0.00,(P=1.000)$ \\
\hline$\geq 5$ & $17(77.3 \%)$ & $14(63.6 \%)$ & & $17(77.3 \%)$ & $17(77.3 \%)$ & \\
\hline $\begin{array}{l}\mathrm{PSQI}<5 \\
(\text { Mean } \pm \mathrm{SD})\end{array}$ & $3.00 \pm 1.24$ & $3.00 \pm 1.00$ & $\mathrm{t}=0.00(\mathrm{P}=1.000)$ & $3.20 \pm 0.83$ & $4.80 \pm 1.02$ & $\mathrm{t}=3.18(\mathrm{P}=0.035)^{*}$ \\
\hline $\begin{array}{l}\mathrm{PSQI} \geq 5 \\
(\mathrm{Mean} \pm \mathrm{SD})\end{array}$ & $10.64 \pm 3.34$ & $8.82 \pm 2.96$ & $\mathrm{t}=8.51(\mathrm{P}=0.000) * * *$ & $8.47 \pm 2.52$ & $9.17 \pm 2.74$ & $\mathrm{t}=3.43(\mathrm{P}=0.003) * *$ \\
\hline
\end{tabular}

8130 Asian Pacific Journal of Cancer Prevention, Vol 16, 2015 
Table 3. Effects of Massage on BP and Heart Rate

\begin{tabular}{lcccccc}
\hline & \multicolumn{5}{c}{ Massage Group $(\mathrm{N}=22)$} \\
\hline & $\begin{array}{c}\text { Before massage } \\
(\text { Mean } \pm \text { SD) }\end{array}$ & $\begin{array}{c}\text { After massage } \\
(\text { Mean } \pm \text { SD) }\end{array}$ & Significance & Before massage & After massage & Significance \\
& $118.31 \pm 13.93$ & $114.95 \pm 13.22$ & $\mathrm{t}=11.27(\mathrm{P}=0000) * *$ & $117.31 \pm 11.87$ & $114.31 \pm 12.68$ & $\mathrm{t}=5.65(\mathrm{P}=0000) * *$ \\
\hline SBP & $73.27 \pm 8.44$ & $72.00 \pm 8.72$ & $\mathrm{t}=3.13(\mathrm{P}=0.005) *$ & $73.27 \pm 6.82$ & $71.50 \pm 6.67$ & $\mathrm{t}=3.93(\mathrm{P}=0.001) *$ \\
DBP & $76.09 \pm 7.87$ & $74.31 \pm 7.74$ & $\mathrm{t}=6.75(\mathrm{P}=0.005) *$ & $74.77 \pm 7.83$ & $72.77 \pm 7.62$ & $\mathrm{t}=7.37(\mathrm{P}=0.000) * *$ \\
HR & & & & & &
\end{tabular}

$* \mathrm{P}<0.01 * * \mathrm{P}<0.001$.

in the intervention group shifted from the moderate to the low level anxiety group because their anxiety scores were decreased after massage, although this decrease was not statistically significant. In addition to this, the mean STAI-s score significantly decreased from 49.68 to 45.68 in the massage group $(\mathrm{P}<0.001)$. Regarding the control group, mean STAI-s scores of caregivers with low level anxiety and moderate level anxiety slightly increased while mean STAI-s scores of caregivers with higher anxiety showed a tendency to decrease. However, these changes in the control group were not significant.

In the massage group, plasma cortisol levels significantly decreased while in the control group the levels slightly increased without creating a significant difference.

There were significant differences in BP values before and after massage with a drop of $3.36 \mathrm{mmHg}$ in mean systolic blood pressure (SBP) and drop of $1.27 \mathrm{mmHg}$ in mean diastolic blood pressure (DBP) on day 1; a drop of $3 \mathrm{mmHg}$ in SBP and a drop of $1.77 \mathrm{mmHg}$ in DBP on day 7. HR was also significantly decreased after massage on day $1(\mathrm{P}<0.01)$ and day $7(\mathrm{P}<0.001)$.

The results of our study showed that $77.3 \%$ of both the massage and control group had poor sleep quality. At the final evaluation, $17.6 \%(n=3)$ of the caregivers with PSQL $\geq 5$ in the intervention group shifted into the PSQL $<5$ group, although this decrease was not statistically significant. However, the mean sleep quality score in caregivers with PSQL $\geq 5$ significantly decreased from 10.64 to 8.82 after massage $(\mathrm{P}<0.001)$. In the control group, the number of patients with PSQL $<5$ and PSQL $\geq 5$ did not change, however, the mean sleep quality scores increased significantly.

\section{Discussion}

The results of the current study supported the positive effects of massage on state anxiety, cortisol level, systolic/ diastolic BP, HR, and sleep quality in caregivers of cancer patients.

Effects of massage on state anxiety: In the current study, anxiety scores decreased significantly in the massage group. This expected finding was also supported by earlier studies.

Previous studies of massage for healthy females (Noto et al., 2010; Kunikata et al., 2012), nurses (Engen et al., 2012), older adults (Harris and Richards, 2010), congestive heart failure patients (Chen et al., 2013), and cancer patients (Wilkinson et al., 2008) have found consistent benefits for anxiety. These findings were also supported by an earlier systematic review (Moyer et al., 2011).

There are only three studies testing the effects of massage on anxiety in caregivers of patients and two of them were on caregivers of cancer patients as far as the authors' present knowledge extends.

In Iwasaki's study (2005), 68 women with hospitalized children received hand and shoulder massage for 20 minutes using essential rose oil and compared 20 mothers with healthy children. After massage, tension-anxiety in mothers with hospitalized children decreased significantly.

In a study conducted by Rexilius et al. (2002), 33 caregivers of patients undergoing autologous hematopoietic stem cell transplant were divided into a massage, a healing touch and a control group. For each subject, massage on face, neck, shoulder and back was carried out for 30 minutes twice per week for 3 weeks. At the end of the study, only the massage group showed significant declines in anxiety scores (Rexilius et al., 2002). And finally, in Goodfellow's study (2003), 42 spouses of patients with cancer were randomly assigned to either the therapeutic back massage or the control group. The massage group received a 20 -minute massage. The result of this study indicated positive effects of massage on mood and perceived stress.

Our results, showing massage was effective to decrease state anxiety, contribute to the collective findings from previous studies.

Effects of massage on cortisol levels: It is well known that cortisol is significantly increased by psychological stressors. Although STAI is a well recognized subjective anxiety assessment tool, subjective psychological tests such as STAI might have limited value if subjects fail to answer reliably. Thus assessing psychosocial status by using stress hormones such as cortisol levels would be much more objective and reliable. Therefore, we assessed serum cortisol levels as an objective indicator of stress or distress, besides assessment of subjective state anxiety by using STAI-s.

In our study, plasma cortisol levels in the intervention group were significantly decreased after massage. This result indicates that the sympathetic function of the autonomic nervous system is suppressed by massage activities although previous studies are not in agreement with the existence or magnitude of such a cortisol reduction induced by massage. For example, Noto et al. (2010) reported that salivary cortisol levels did not change after back massage in young healthy females. In 
Moyer et al. study (2011), six previous reviews examining massage's effect on cortisol have been reviewed. They found that the effect of massage on cortisol levels was very small or nonsignificant; however, when massage is applied in multiple doses, the effect would be more significant.

In this study, a decrease in cortisol levels after massage was not surprising because massage was applied in multiple doses as 15 minutes every day for one week, thus sustaining the effect of massage might be effective to decrease cortisol levels, as mentioned by Moyer et al. (2011).A decrease in serum cortisol levels after massage, in our study, suggests that massage has effective to reduce stress.

Effects of massage on BP and HR: Our findings showed that both BP and HR, which are a physiological indicators, significantly decreased by massage in the intervention group as expected.

Our finding on BP was consistent with the results of previous studies although most of these studies were conducted in hypertensive patients (Supa'at et al., 2014; Xiong et al., 2015). The one of the previous study in congestive heart failure patients (Chen et al., 2013) also showed significant decrease in BP after massage. Regarding HR, all previous studies (Noto et al., 2010; Chen et al., 2013, Supa'at et al., 2013) indicated that massage has a decreasing effect on HR.

Even though the subjects involved and the methods and duration of massage are different from other studies, our results are consistent with previous studies showing that massage is effective to decrease BP and HR. The reduction in BP and HR could be explained by a comfortable sensation and relaxation, as well as a change from sympathetic activities to parasympathetic activities and finally an increase of parasympathetic activities induced by massage as shown by Ouchi et al. (2006).

Effects of massage on sleep quality: In this study, the PSQI scores of $77.3 \%$ of the caregivers in both the massage and control group were above 5 , which indicated poor sleep quality. Sleep is essential for optimum health and HRQOL. In addition, impaired or inadequate sleep is associated with health related morbidity including physical fatigue, depressive symptoms (Carter and Acton, 2006); increased level of stress (Pollock et al., 2013), poor physical health (Northouse et al., 2012), and poor quality of life (Perlis et al., 2014). Thus, targeted sleep-improving interventions such as massage to improve health and quality of life should be considered.

In the current study, massage was effective to improve sleep quality. After massage, the percentages of caregivers with poor sleep quality decreased from $77.3 \%$ to $63.6 \%$ with a decrease of $13.7 \%$. Mean PSQL scores for caregivers with PSQL $\geq 5$ was also significantly decreased by massage. Similar to our result, MacDonald (1998) showed that sleep quality increased in primary family caregivers after neck, shoulder and back massage; in addition, caregivers reported reduced physical and emotional stress after massage. In a study by Mackereth et al. (2005), it was also reported that massage therapy caused improvement in sleep duration. Thus, previous literature supports our finding that massage improves sleep.

Massage, as shown in current and earlier studies, promotes a significant decrease in cortisol levels, promotes parasympathetic activation, which causes reductions in BP and HR (Moyer et al., 2004; Liao et al., 2014), anxiety, stress, and depression (Moyer et al., 2004). All of these factors might have a contribution on sleep quality.

This study has two main limitations that should be acknowledged. First, the sample size was relatively small although demographic data in massage and control groups were similar. Second, we measured plasma cortisol rather than salivary cortisol. Although we know that plasma and salivary cortisol levels are comparable, there is doubt that injection pain induced by blood sampling, in this study, may have increased cortisol levels.

Despite these limitations, this study has several strengths. A blocked randomised controlled design allows greater power with smaller sample sizes and eliminates heterogeneity between the intervention and control groups. In addition, the small sized sample in this study is still comparable to previous studies of caregivers for oncology patients (Rexilius et al., 2002; Goodfellow, 2003; Mackereth et al., 2005). Thus, we can conclude that our results can be used for comparative purposes.

In conclusion, this study has shown that back massage was effective in reducing state anxiety, cortisol levels, BP and HR, as well as increasing sleep quality in caregivers for patients with cancer. Into a busy oncology setting, oncology nurses can take advantage of this complementary, non-pharmacologic and easily implemented method, as an independent nursing action to family support caregivers for patients with cancer.

\section{References}

Agargun MY, Kara H, Anlar O (1996). Validity and reliability of pittsburgh sleep quality index. Turk Psikiyatri Derg, 7, 107-15.

Butow PN, Price MA, Bell ML, et al (2014). Caring for women with ovarian cancer in the last year of life: a longitudinal study of caregiver quality of life, distress and unmet needs. Gynecol Oncol, 132, 690-7.

Buysse DJ, Reynolds CF, Monk TH, et al (1989). The pittsburgh sleep quality index: a new instrument for psychiatric practice and research. Psychiatry Res, 28, 193-213.

Carter PA, Acton GJ (2006). Personality and coping: predictors of depression and sleep problems among caregivers of individuals who have cancer. J Gerontol Nurs, 32, 45-53.

Chen WL, Liu GJ, Yeh SH, et al (2013). Effect of back massage intervention on anxiety, comfort, and physiologic responses in patients with congestive heart failure. J Altern Complement Med, 19, 464-70.

Cora A, Partinico M, Munafò M, Palomba D (2012). Health risk factors in caregivers of terminal cancer patients: a pilot study. Cancer Nurs, 35, 38-47.

Engen DJ, Wahner-Roedler DL, Vincent A, et al (2012). Feasibility and effect of chair massage offered to nurses during work hours on stress-related symptoms: a pilot study. Complement Ther Clin Pract, 18, 212-5.

Field T, Hernandez-Reif M, Diego M, Schanberg S, Kuhn C (2005). Cortisol decreases and serotonin and dopamine increase following massage therapy. Int J Neurosci, 115, 1397-413.

Given B, Sherwood PR (2006). Family care for the older person with cancer. Semin Oncol Nurs, 22, 43-50.

Goodfellow LM (2003). The effects of therapeutic back massage 
Back Massage Decreases Anxiety, Blood Pressure, Heart Rate and Increases Sleep Quality in Cancer Patient Caregivers on psychophysiologic variables and immune function in spouses of patients with cancer. Nurs Res, 52, 318-28.

Harris M, Richards KC (2010). The physiological and psychological effects of slow-stroke back massage and hand massage on relaxation in older people. J Clin Nurs, 19, 917-26.

Iwasaki M (2005). Intervational study on fatigue relief in mothers caring for hospitalized children: effect of massage incorporating techniques from oriental medicine. Kurume J Med, 52, 19-27.

Kunikata H, Watanabe K, Miyoshi M, Tanioka T (2012). The effects measurement of hand massage by the autonomic activity and psychological indicators. J Med Invest, 59, 206-12.

Lambert SD, Girgis A, Lecathelinais C, Stacey F (2013). Walking a mile in their shoes: anxiety and depression among partners and caregivers of cancer survivors at 6 and 12 months postdiagnosis. Support Care Cancer, 21, 75-85.

Lengacher CA, Kip KE, Barta M, et al (2012). A pilot study evaluating the effect of mindfulness-based stress reduction on psychological status, physical status, salivary cortisol, and interleukin- 6 among advanced-stage cancer patients and their caregivers. J Holist Nurs, 30, 170-85.

Li Q, Loke AY (2013). A spectrum of hidden morbidities among spousal caregivers for patients with cancer, and differences between the genders: a review of the literature. Eur J Oncol Nurs, 17, 578-87.

Liao IC, Chen SL, Wang MY, Tsai PS. (2014). Effects of massage on blood pressure in patients with hypertension and prehypertension: a meta-analysis of randomized controlled trials. J Cardiovasc Nurs, [Epub ahead of print]

Lund L, Ross L, Petersen MA, Groenvold M (2014). Cancer caregiving tasks and consequences and their associations with caregiver status and the caregiver's relationship to the patient: a survey. BMC Cancer, $\mathbf{1 4}, 541$.

MacDonald G (1998). Massage as a respite intervention for primary caregivers. Am J Hosp Palliat Care, 15, 43-7.

Mackereth P, Sylt P, Weinberg A, Campbell G (2005). Chair massage for carers in an acute cancer hospital. EJON, $\mathbf{9}$, 167-79.

Mitchell AJ, Ferguson DW, Gill J, Paul J, Symonds P (2013). Depression and anxiety in long-term cancer survivors compared with spouses and healthy controls: a systematic review and meta-analysis. Lancet Oncol, 14, 721-32.

Morris BA, Thorndike FP, Ritterband LM, et al (2015). Sleep disturbance in cancer patients and caregivers who contact telephone-based help services. Support Cancer Care, 23, 113-20.

Moyer CA, Rounds J, Hannum JW (2004). A meta-analysis of massage therapy research. Psychol Bull, 130, 3-18.

Moyer CA, Seefeldt L, Mann ES, Jackley LM (2011). Does massage therapy reduce cortisol? a comprehensive quantitative review. J Bodyw Mov Ther, 15, 3-14.

Northouse L, Williams AL, Given B, McCorkle R (2012). Psychosocial care for family caregivers of patients with cancer. J Clin Oncol, 30, 1227-34.

Noto Y, Kudo M, Hirota K (2010). Back massage therapy promotes psychological relaxation and an increase in salivary chromogranin A release. J Anesth, 24, 955-8.

Oucki Y, Kanno T, Okada H, et al (2006). Changes in cerebral blood flow under the prone condition with and without massage. Neurosci Lett, 407, 131-5.

Oner N, Le Compte A (1985). The Handbook for State-trait Anxiety Inventory. Istanbul: Bosphorus University Publications, No: 333.

Palos GR, Mendoza TR, Liao KP, et al (2011). Caregiver symptom burden: the risk of caring for an underserved 